\title{
Comportamiento alimentario: Revisión conceptual
}

\author{
Feeding behavior: a conceptual review
}

Arlyn Torres Meza ${ }^{a}$, Jesús Cisneros-Herrera ${ }^{b}$ y Gelacio Guzmán-Díaz ${ }^{c}$

\begin{abstract}
:
Studying feeding behavior implies several disciplines, being psychology one of them. Feeding behavior refers to a set of actions performed as a response to a biological, psychological and socio-cultural motivation related to food intake. This review presents a set of antecedents and definitions of feeding behavior in order to separate it from feeding conduct. Moreover, the so-called risk feeding behaviors and feeding disorders are reviewed. At the end, some studies related do feeding behavior in student community are presented, as well as some researches about feeding behavior during the pandemics caused by COVID-19.
\end{abstract}

Keywords:

Feeding behavior, risk feeding behavior, feeding disorders.

Resumen:

El comportamiento alimentario es un tema que implica distintas disciplinas para su estudio, una de ellas es la psicología. La conducta alimentaria se trata de un conjunto de acciones ejecutadas en respuesta a una motivación biológica, psicológica y sociocultural, relacionada con la ingestión de alimentos. La presente revisión presenta una serie de antecedentes y definiciones del comportamiento alimentario con el fin de distinguirlo de la conducta alimentario. Así mismo se revisan los llamadas comportamientos alimentarios de riesgo y los trastornos del comportamiento alimentario. Finalmente se presenta algunos estudios relativos al comportamiento alimentario en la comunidad estudiantil y algunas investigaciones sobre el comportamiento alimentario en tiempos de pandemia por COVID-19.

Palabras Clave:

Comportamiento alimentario, Comportamientos alimentarios de riesgo, Trastornos del comportamiento alimentario.

\section{Comportamiento Alimentario}

\section{Antecedentes}

El comportamiento alimentario se ha establecido desde un estudio multidisciplinario, que requiere la colaboración de distintas perspectivas para su investigación.

En 1912, dos publicaciones establecieron la investigación en este tema, enfocándose principalmente en el hambre: An explanation of hunger de Walter B. Cannon y A. L. Washburn, y Orígenes del conocimiento: El hambre de Ramón Turró Darder. La publicación de Cannon abordando el tema desde un enfoque fisiológico, mientras que Turró desde el aspecto filosófico y psicológico.

Cannon y Washburn, citados por Martínez, LópezEspinoza y Díaz $(2007)^{2}$, establecen que las contracciones estomacales y el contenido gástrico se relacionan con las sensaciones del hambre, siendo estas las que iniciaban el acto de comer. Igualmente, Cannon aporta su teoría homeostática, la homeostasis refiere a un proceso regulatorio en los organismos para mantener su equilibrio. Por lo que la conducta de comer inicia como respuesta a un déficit de alimento, es un proceso de retroalimentación negativa.

Turró (1912) en Orígenes del conocimiento: El hambre, establece que, el hambre responde a la necesidad de reparar las pérdidas del organismo incorporando las sustancias alimenticias al medio interno; las sustancias pasan por trasformaciones en su composición y en su estructura molecular, debido a que deben ser nutrimentos para formar parte del organismo. El verdadero problema psicofisiológico del hambre consiste en conocer y estudiar el efecto que la causa; para entender al hambre, debe conocerse cuándo y cómo esa sensación aparece, cómo varía de acuerdo a características individuales. La sensación del hambre se reduce a un fenómeno

Autor de Correspondencia, Universidad Autónoma del Estado de Hidalgo, https://orcid.org/0000-0002-3268-0261 Email: to355899@uaeh.edu.mx

${ }^{\mathrm{b}}$ Universidad Autónoma del Estado de Hidalgo, https://orcid.org/0000-0003-1370-8086, Email: jesus_cisneros@uaeh.edu.mx c Universidad Autónoma del Estado de Hidalgo, https://orcid.org/0000-0003-4869-8658, Email: gelacio_guzman@uaeh.edu.mx 
experimental que se apoya en la observación empírica. Desde un enfoque psicológico el hambre es la conciencia de la ausencia de las sustancias nutricias en el organismo. Además, propone la aproximación trófica basada en el aprendizaje.

De acuerdo con López-Espinoza, Martínez, AguileraCervantes y Valdés-Miramontes $(2021)^{1}$, a lo largo de los años siguientes a las dos publicaciones, anteriormente mencionadas, que dieron paso al estudio del comportamiento, se desarrollaron diversas teorías, dentro de la visión biológica se destacan: la hipótesis del factor lipostático regulatorio de Kennedy, la teoría glucostática de Mayer, la teoría termostática de Brobeck, y la teoría neuro-endócrina estudiada por Blundell, Latham y Wurtman. Mientras que desde la perspectiva psicológica se retoma a exponentes reconocidos como, Watson que establece la conducta como objeto de estudio de la psicología, Pavlov con la teoría del condicionamiento pavloviano, y Skinner con la teoría del condicionamiento operante. Otras aportaciones significativas son: la conducta como mecanismo regulador alimentario de Richter, la externalización del drive de Anderson, los determinantes de la conducta de Young, y los factores temporales en alimentación propuestos por Bolles, Timberlake y Lucas.

En la investigación de la conducta alimentaria, se establecen tres puntos importantes para su estudio, el primero de ellos establece que la conducta es un mecanismo que permite influir en la conducta de otros, por ejemplo, la forma en la que los adultos de una familia se alimentan un es determinante en la cantidad, calidad y forma de la alimentación de los infantes. El segundo punto trata sobre la conducta como un regulador del estado interno de los organismos. Mientras que el tercer punto señala que la investigación en la conducta alimentaria es una aproximación para mejorar la situación actual en el campo de la alimentación mundial, puesto que se trata del resultado del proceso evolutivo ${ }^{4}$.

\section{Definiciones}

López-Espinoza, Martínez-Moreno, Aguilera-Cervantes, Valdés-Miramontes et al. $(2018)^{4}$, nombran como comportamiento alimentario al conjunto de conocimientos dirigidos a la alimentación, siendo éstos, una visión multidisciplinaria compuesta de diversas áreas científicas. Es decir, refieren a toda acción o estrategia, dirigida a lo que hacemos para alimentarnos como la definición del comportamiento alimentario. Dentro del comportamiento alimentario se reconocen diferentes tipos de conductas alimentarias como unidades funcionales de los distintos procesos que integran al comportamiento alimentario, por ejemplo las preferencias, los hábitos, la selección y las formas en que se establece la ingesta.
Además, se establece una diferencia entre comportamiento alimentario y conducta alimentaria; el primer concepto mencionado, es la serie de acciones dirigidos a dar, proveer y producir alimentos; mientras que la conducta alimentaria es el acto de ingerir, consumir, deglutir, etc., en una temporalidad especifica ${ }^{4}$.

Aunque se plantea una distinción entre ambos conceptos, otros autores como los siguientes, realizan una definición conjunta, haciendo referencia al comportamiento alimentario y conducta alimentaria como sinónimos.

Para Saucedo-Molina y Unikel $(2010)^{5}$, la conducta alimentaria, si bien se asocia inmediatamente con la ingestión de alimentos, es una conducta intervenida por factores externos al individuo y no se relaciona al solo hecho de satisfacer las necesidades de alimentación y sus exigencias nutricias. Por lo que, la conducta alimentaria es entendida como un conjunto de acciones ejecutadas en respuesta a una motivación biológica, psicológica y sociocultural, relacionada con la ingestión de alimentos. Vargas y Aguilar citados por Saucedo $(2003)^{6}$, describen la conducta alimentaria como un fenómeno en el que actúan múltiples factores como, los biológicos (hambresaciedad, sed, estados fisiológicos), los factores geográficos (localización geográfica, clima, disponibilidad de alimentos), los psicológicos (preferencias gustos, creencias) y los socioculturales (costumbres, tradiciones, nivel económico y cultura).

De una forma simple, Osorio, Weisstaub y Castillo $(2002)^{7}$ definen la conducta alimentaria como "el comportamiento normal relacionado con los hábitos de alimentación, la selección de alimentos que se ingieren, las preparaciones culinarias y las cantidades ingeridas de ellos".

\section{Perspectivas de estudio}

De acuerdo con Santacoloma y Quiroga $(2009)^{8}$, la conducta alimentaria puede ser estudiada desde diferentes perspectivas como la biológica y la psicológica (variables cognitivas y el análisis del comportamiento), que permiten conocer la adquisición, establecimiento, mantenimiento y extinción de la misma. La perspectiva biológica y las variables cognoscitivas describen lo que ocurre cuando un organismo emite la conducta alimentaria, mientras que, el análisis del comportamiento informa acerca de las variables ambientales involucradas en la ingesta de alimentos.

En la perspectiva biológica, la regulación y alteración de la conducta alimentaria se encuentran relacionadas con variables de tipo biológico y fisiológico. La ingesta de alimentos es intervenida por la región lateral del hipotálamo (conocida como el centro del apetito), la región ventromedial (centro de la saciedad), y hormonas como la 
grelina (hormona del apetito) y la leptina (hormona de la saciedad). Además, los factores neurosensoriales como la visión, el olfato y el gusto, también tienen presencia en el proceso alimenticio ${ }^{9}$.

Mientras que la perspectiva psicológica incluye dos enfoques, el primero de ellos es el cognoscitivo donde se incluyen variables mentales y relacionadas con los procesos psicológicos. El segundo enfoque es el análisis del comportamiento.

Por otro lado, el enfoque cognoscitivo describe los procesos de información y la existencia de estructuras cognitivas que infieren en la producción y emisión del comportamiento alimentario; realiza una consolidación de los procesos y estructuras cognoscitivas, que influyen en conductas alimenticias creando hábitos de alimentación. De acuerdo con esta perspectiva, la conducta alimentaria está determinada por variables mentales; por ejemplo, en el consumo de alimentos interfiere el estado de ánimo y las emociones, debido a que se ha demostrado que frecuentemente una persona disminuye la cantidad de alimento que consume al experimentar emociones negativas ${ }^{8}$.

Una variable cognoscitiva que se ha estudiado en relación con al comportamiento alimentario es el estrés. GonzálezTorres, Carranza y Reyes-Huerta $(2018)^{10}$, mencionan que el estrés, moderado o severo, con frecuencia disminuye la ingesta de alimentos y el peso corporal, este fenómeno siendo congruente con los cambios fisiológicos producidos como respuesta del estrés.

El análisis del comportamiento alimentario permite establecer medidas como la presencia o ausencia de la comida, la frecuencia del consumo de alimentos durante cierta cantidad de tiempo, cantidad de alimento dentro de un periodo, duración de la comida, entre otras medidas, además de estudiar variables ambientales que afectan el comportamiento en la búsqueda, la elección y el consumo de alimentos ${ }^{8}$.

\section{Conductas alimentarias de riesgo (CAR)}

Saucedo-Molina y Unikel $(2010)^{5}$ establecen que las alteraciones en la conducta alimentaria dan origen a las llamadas conductas alimentarias de riesgo (CAR) y por supuesto, a los trastornos de la conducta alimentaria (TCA).

Las conductas alimentarias de riesgo (CAR) se tratan de actitudes encaminadas a obtener o mantener una figura corporal delgada. Tanto en la adolescencia como en la adultez joven, estas conductas tienen mayor frecuencia, debido a los cambios físicos y psicológicos de estas etapas, y a la importancia que se le otorga a la imagen corporal. Las CAR se vinculan con la aparición de los trastornos de la conducta alimentaria (Shisslak et al., 1995; Sparti et al., 2019; y Loth et al., 2015; citados por
Villalobos, Unikel, Hernández-Serrato \& Bojórquez, 2020) $)^{11}$.

La alteración persistente de la alimentación y en el comportamiento alimentario son lo que caracterizan a los trastornos de la conducta alimentaria y de la ingesta de alimentos; en el Manual Diagnóstico y Estadístico de los Trastornos Mentales se incluyen: pica, trastorno por rumiación, trastorno de evitación/restricción de la ingesta de alimentos, anorexia nerviosa, bulimia nerviosa, trastorno de atracones y otros trastornos de la conducta alimentaria 0 de la ingesta de alimentos ${ }^{12}$. De forma general, los trastornos de la conducta alimentaria (TCA) tienen criterios diagnósticos como intenso deseo de adelgazar, prácticas destinadas a este fin, la consideración por parte del sujeto de que una figura corporal delgada es indispensable para la autoestima ${ }^{13}$. La causa especifica de los TCA es desconocida, sin embargo, interviene factores genéticos, biológicos, psicológicos, familiares y socioculturales en su desarrollo. Los factores socioculturales como la promoción de modelos estéticos corporales y de la delgadez son los más importantes ${ }^{14}$.

En síntesis, las conductas alimentarias de riesgo son manifestaciones similares a los trastornos de la conducta alimentaria, pero de frecuencia e intensidad menor. La práctica del ayuno, los atracones, el seguimiento de dietas restrictivas, el vómito auto-inducido, y el uso de laxantes y diuréticos, son considerados algunos ejemplos de conductas de riesgo ${ }^{15}$.

Dentro de las CAR, el ayuno es entendido como la abstinencia a los alimentos y líquidos durante un tiempo definido. A largo plazo, es causa de daños en el sistema inmunológico, infecciones en riñones e hígado, ulceras perforadas y deficiencia pulmonar cardiaca; mientras que a nivel psicológico provoca disociaciones cognitivas, limitando la habilidad para realizar tareas cotidianas, además de consecuencias en la concentración, juicio y en los signos de alerta (Maughan, Fallah \& Coyle, 2010; y Toro \& Castro, 2004 citados por Ortega-Luyando et al., 2018) ${ }^{16}$.

Otra conducta alimentaria de riesgo es el atracón, que implica una pérdida parcial o total del control sobre la alimentación, es decir, se trata de la alimentación descontrolada. Las personas que realizan esta práctica consumen una numerosa cantidad de alimentos durante un periodo corto, acompañado de la sensación de falta de control, por lo que también suele ser utilizado el término de alimentación compulsiva para hacer referencia a esta conducta $^{17}$.

Con el propósito de controlar el peso y el mejoramiento de la figura corporal, puede presentarse la dieta restrictiva, que es la tendencia repetitiva de la propia privación de alimentos que una persona considera que lo harán engordar, además de incluir la eliminación del desayuno, 
la comida y/o la cena a lo largo del día (Gómez PérezMitré \& Ávila, 1998; y Huon \& Strong, 1998; citados por Saucedo, 2003) ${ }^{6}$.

Mientras que, el vómito auto-inducido es la acción intencional de realizar contracciones estomacales por medio de la autoestimulación del paladar o un área cercana, con el propósito de desechar el contenido gástrico por la boca. Algunas consecuencias de esta práctica son daños en el esófago, la erosión dental y las úlceras en la boca ${ }^{16}$.

Finalmente, en los intentos por perder peso rápidamente, el uso de diuréticos y laxantes hacen su presencia. Los diuréticos corresponden a un grupo de medicamentos que se emplean para ajustar el volumen o la composición de los líquidos del organismo, estimulando la actividad renal y aumentando la emisión de la orina, por lo que su uso sin prescripción o indicación médica puede generar un desequilibrio electrolítico. Asimismo, los laxantes son medicamentos aceleran la eliminación fecal estimulando la actividad intestinal, el abuso excesivo de estos fármacos son causa de cólicos abdominales. ${ }^{18} 16$

\section{Relación entre el comportamiento alimentario y otras variables}

Adicional a las variables biológicas y las psicológicas que se abordan en el estudio e investigación del comportamiento alimentario, pueden interferir otras como las sociales y culturales.

Las personas establecen hábitos de consumo y patrones de comportamiento influidos por variables culturales y sociales, tal como la promoción de delgadez y los ideales de belleza a alcanzar, propuestos por una cultura o sociedad en un espacio y temporalidad determinada, y distribuidos por la publicidad y los medios masivos de comunicación ${ }^{8}$.

En el desarrollo de conductas alimentarias de riesgo, la baja autoestima y la imagen corporal son factores de gran impacto $^{15}$.

La baja autoestima hace referencia a las personas que experimentan un sentimiento de inferioridad y timidez en sus relaciones interpersonales, causado por la inseguridad, desconfianza de sus facultades y la necesidad de aprobación debido a que tiene distintos complejos $^{19}$.

Santacoloma y Quiroga $(2009)^{8}$ establecen que la autoestima es una variable que puede verse alterada con los estándares de belleza, por lo que, quienes consideran que no se acercan a lo establecido, padecen baja autoestima, pudiendo detonar una alteración en la conducta alimentaria.

Asimismo, Lameiras, Calado, Rodríguez y Fernández $(2003)^{20}$ definen la imagen corporal como una representación mental de varios aspectos de la apariencia física, lograda a partir del componente perceptivo, el cognitivo- afectivo y el conductual; al verse afectada produce una insatisfacción causando desequilibrio y perturbación entre los componentes, y provocando que la persona pueda involucrarse en dietas dañinas.

\section{Exploración empírica: comportamiento alimentario y población estudiantil}

Saucedo-Molina y Unikel $(2010)^{5}$ plantean que los factores de riesgo más estudiados de los TCA, son: las conductas alimentarias, el Índice de Masa Corporal (IMC) y las actitudes hacia el cuerpo provenientes de la interiorización de una figura corporal delgada. Por lo que realizaron una investigación que tuvo por objetivo conocer la prevalencia de conductas alimentarias de riesgo en estudiantes de preparatoria y universidad, además de la relación que estas conductas tienen con la interiorización del ideal estético de delgadez y con el Índice de Masa Corporal (IMC), dirigido a estudiantes de ambos sexos en una escuela privada de Pachuca, Hidalgo. Se trabajó con una muestra compuesta por 845 sujetos, siendo 381 hombres y 464 mujeres, el $45.1 \%$ de nivel preparatoria de 15 a 17 años y $54.9 \%$ de licenciatura de 18 a 23 años. Para su investigación, utilizaron el Cuestionario Breve de Conductas Alimentarias de Riesgo (CBCAR), donde se realizan preguntas sobre las alteraciones alimentarias convenientes de los TCA de acuerdo con los criterios diagnósticos del DSM-IV, y el Cuestionario de Actitudes hacia la Figura Corporal, además del IMC que se obtuvo con el peso y la estatura de cada sujeto. Los resultados que se obtuvieron indican que la prevalencia de las CAR fue de $8.4 \%$ en mujeres y $2.9 \%$. en hombres; en las mujeres, los ayunos, dietas y uso de diuréticos fue mayor en las estudiantes de licenciatura, mientras que la preocupación por engordar, los atracones, falta de control al comer, ejercicio excesivo, vómito autoinducido, uso de laxantes y de pastillas fue mayor en estudiantes de preparatoria. De las mujeres con CAR elevadas, el $84.2 \%$ de preparatoria y el $80 \%$ de licenciatura tuvieron altas puntuaciones en el Cuestionario de actitudes hacia la figura corporal. y en los hombres fueron 33.3\% y $62.5 \%$ respectivamente. De acuerdo con los resultados obtenidos indicaron que la interiorización del ideal estético de delgadez y el sexo, tuvieron asociación con las CAR, sin embargo, el IMC y el nivel de estudios no tuvieron una asociación significativa; concluyeron en la población estudiada están presentes las CAR y que se encuentran principalmente en mujeres.

Franco, Martínez et al. $(2010)^{22}$ realizaron una investigación sobre la ocurrencia de las conductas alimentarias de riesgo y la sintomatología de los trastornos de la conducta alimentaria, centrándose en estudiantes universitarios de del Sur de Jalisco, México. Participó una 
muestra de 252 participantes, 143 mujeres y 109 hombres pertenecientes a una universidad pública del Jalisco, México; a quienes se les aplico el Cuestionario de Actitudes Alimentarias (EAT-40), Cuestionario de Bulimia de Edimburgo (BITE), Cuestionario de Imagen Corporal (BSQ). Los participantes se agruparon de acuerdo a su índice de masa corporal y peso en mujeres fue: $11.2 \%$ bajo peso, $66.4 \%$ peso normal, $18.2 \%$ sobrepeso y $4.2 \%$ obesidad; por su parte en los hombres la distribución fue: $4.6 \%$ bajo peso, $61.5 \%$ peso normal, $23.8 \%$ sobrepeso y $10.1 \%$ obesidad. Los resultados indicaron que las conductas de riesgo que se presentan con un porcentaje mayor en mujeres, son las dietas restrictivas y el empleo de productos para adelgazar, mientras que en varones es la práctica de ejercicio para quemar calorías. La insatisfacción corporal se presentó en el 14\% de las mujeres y en el $10 \%$ de los hombres. Asimismo, se identificaron la cantidad de conductas de riesgo en los participantes con insatisfacción corporal: en las mujeres con peso normal fue entre una y tres conductas, con sobrepeso hasta cinco conductas y con obesidad dos o tres conductas, mientras que, en hombres, hasta dos conductas de riesgo los situados en peso normal, dos o tres conductas en sobrepeso y dos conductas en los que tenían obesidad. Concluyeron que existe una mayor presencia de conductas alimentarias de riesgo, insatisfacción corporal y sintomatología de TCA mayormente en las mujeres que en hombres; además su estudio confirmo que las conductas alimentarias de riesgo y la sintomatología de TCA igualmente se presentan en ciudades medianas con desarrollo económico medio.

Ramón, Martínez et al. (2019)22 elaboraron un estudio descriptivo-transversal, ejecutando una evaluación entre la calidad de la dieta, las dimensiones de la salud psicológica y el sueño; su investigación aborda la conducta alimentaria y cómo esta se relaciona con distintas variables como el estrés, la ansiedad, la depresión y el insomnio en estudiantes de la Universidad San Jorge en Zaragoza, España. Se plantearon que existe evidencia que vincula la alimentación con la salud psicológica y que esa relación no se había explorado lo necesario en estudiantes universitarios. La investigación la realizaron bajo los resultados obtenidos de, la versión breve del International Physical Activity Questionnaire (IPAQ breve) en su versión en español, el Índice de Alimentación Saludable (IAS), la escala breve de Depresión, Ansiedad y Estrés (DASS-21) y el Índice de Severidad del Insomnio. Participaron 1055 estudiantes en un rango de edad de 18 a 42 años, de ellos, el 82,3\% presentaron una alimentación necesitada de cambios o poco saludable, donde las mujeres mostraron una alimentación menos saludable que los hombres; los niveles de ansiedad, depresión y estrés se presentan en el $23,5 \%, 18,6 \%$ y $33,9 \%$, respectivamente, de los participantes; asimismo el $43,1 \%$ de los universitarios presentaban insomnio en alguna de sus categorías. Concluyeron, de acuerdo con sus resultados, que hay una prevalencia elevada de estrés, ansiedad, depresión e insomnio entre los universitarios, mayor en las mujeres que en los varones.

Una investigación reciente, es la de Serin y Can Koç $(2020)^{23}$, quienes elaboraron una examinación de las conductas alimentarias y los estados depresivos de universitarios que han estado en sus hogares durante la pandemia por COVID-19. La muestra se integró por 1064 estudiantes, 440 hombres y 624 mujeres. La investigación se elaboró con la utilización del Cuestionario Holandés de Conducta Alimentaria (DEBQ) que realiza una evaluación de diferentes sub-dimensiones, donde se consideran las conductas alimentarias emocionales, las conductas alimentarias externas y las conductas alimentarias restringidas; y la Escala de Depresión del Centro para Estudios Epidemiológicos (CES-D) que evalúa los síntomas depresivos de los estudiantes. Se encontró una diferencia significativa en todas las variables, incluida la edad, el género, el autoaislamiento, la actividad física regular en el hogar durante el COVID-19 y antes del brote de la pandemia. Además, en la correlación entre las puntuaciones de la escala de conducta alimentaria y las puntuaciones de la escala de depresión, no se encontró una relación significativa entre la alimentación externa y la depresión.

\section{Comportamiento Alimentario y el confinamiento por la pandemia por COVID-19}

Tazeoglu, Bozdogan, e Idiz (2021) ${ }^{24}$ establecen que el acto de comer es la necesidad más importante para sobrevivir, se trata de una necesidad biológica que también tiene una perspectiva psicológica. De acuerdo con su estudio, donde realizan una evaluación de la conducta alimentaria de los estudiantes de la Universidad Osmaniye Korkut Ata durante el periodo de cuarentena por el COVID-19, la alimentación restringida y la alimentación emocional han aumentado significativamente durante el periodo posterior al brote de la pandemia, siendo el estrés una variable importante en desencadenar un comportamiento compulsivo, además de ser un factor con efectos psicosociales que interviene en las elecciones del consumo de alimentos, ellos plantean que el consumo de alimentos ricos en grasas y azucarados puede aumentar la obesidad cuando las personas intentan reprimir sus emociones en momentos de estrés.

Almendra-Pegueros, Baladia et al. $(2021)^{26}$ de acuerdo con un análisis de la conducta alimentaria a través de la restricción dietética, alimentación emocional e ingesta incontrolada, y los factores que se asocian con estas 
conductas en adultos durante el confinamiento por COVID-19 en países hispanohablantes, plantean que el confinamiento ha producido modificaciones significativas, crenado patrones que no son saludables, además de una reducción de la actividad física y el incremento del sedentarismo, trayendo consigo consecuencias en el estado conductual y emocional: la depresión, ansiedad, estrés e irritabilidad, y su relación con la nutrición, los hábitos del sueño y de actividad física; además es necesario considerar que estas modificaciones han incrementado en el grupo de edad de 18 a 34 años de edad.

Sin embargo, Pérez-Rodrigo, Gianzo, Hervás et al. $(2020)^{26}$ los cambios de hábitos alimenticios tienen una mayor tendencia al consumo de alimentos saludables, un descenso en el consumo de alimentos de menor interés nutricional y el aumento de la práctica de cocinar en casa durante el confinamiento por la pandemia por COVID-19. Sin embargo, no se descartan las diferencias individuales en la modificación de hábitos de consumo alimentario, con respecto al sexo en función de la edad, si son personas que viven el confinamiento a solas, con sus familias, pareja o con otras personas.

\section{Referencias}

[1] López-Espinoza, A., Martínez, A. G., Aguilera-Cervantes, V. G., \& Valdés-Miramontes, E. H. (2021). Alimentación, integración y cambio de perspectiva. Retos futuros del comportamiento alimentario. Journal $\begin{array}{llll}\text { of Behavior and Feeding, 1(1), 96-102. } & \end{array}$ http://www.jbf.cusur.udg.mx/index.php/JBF/article/view/19

[2] Martínez, A. G., López-Espinoza, \& Díaz, F. (2007). Modelos de regulación en conducta alimentaria. Investigación en Salud, 9(3), 172177. https://www.medigraphic.com/pdfs/invsal/isg-2007/isg073e.pdf

[3] Turró, R. (1912). Orígenes del conocimiento: El hambre. Minerva. https://archive.org/details/B092492/mode/2up

[4] López-Espinoza, A., Martínez-Moreno, A. G., Aguilera-Cervantes, V. G., Valdés-Miramontes, E. H., Santillán-Rivera, M. S., GonzálezMartínez, A. M., \& García-Sánchez, N. E. (2018). Investigación en comportamiento alimentario. Una perspectiva contemporánea. En A. López-Espinoza, A. G. Martínez \& V. G. Aguilera-Cervantes (Coords.), Investigaciones en comportamiento alimentario. Reflexiones, alcances $y$ retos (pp. 1-11) Porrúa. http://www.cusur.udg.mx/es/sites/default/files/investigaciones en com portamento_alimentario.pdf

[5] Saucedo-Molina, T. J., \& Unikel, C. (2010). Conductas alimentarias de riesgo, interiorización del ideal estético de delgadez e índice de masa corporal en estudiantes hidalguenses de preparatoria y licenciatura de una institución privada. Salud Mental, 33(1), 11-19. http://www.scielo.org.mx/scielo.php?script=sci arttext\&pid=S0185$\underline{33252010000100002}$

[6] Saucedo, T. J. (2003). Modelos predictivos de dieta restringida en púberes, hombres y mujeres y en sus madres (Tesis doctoral, Universidad Nacional Autónoma de México). http://132.248.9.195/ppt2002/0316233/Index.html

[7] Osorio, J., Weisstaub, G., \& Castillo, C. (2002). Desarrollo de la conducta alimentaria en la infancia y sus alteraciones. Revista Chilena de Nutrición, 29(3). http://dx.doi.org/10.4067/S0717$\underline{75182002000300002}$

[8] Santacoloma, A. M., \& Quiroga, L. A. (2009). Perspectivas de estudio de la conducta alimentaria. Revista Iberoamericana de Psicología: $\begin{array}{lcccc}\text { Ciencia } & y & \text { Tecnología, } & \text { 2(2), } & \text { 7-15. } \\ \text { https://reviberopsicologia.ibero.edu.co/article/view/rip.2201/314 } & \end{array}$

9] Carranza, L. E. (2016). Fisiología del apetito y el hambre. Enfermería Investiga, Investigación, Vinculación, Docencia y Gestión, 1(3), 117124. https://dialnet.unirioja.es/servlet/articulo? codigo $=6194254$

[10] González-Torres, M. L., Carranza, R., \& Reyes-Huerta, H. E. (2018). El comportamiento alimentario desde los procesos psicológicos. En A. López-Espinoza, A. G. Martínez \& V. G. Aguilera-Cervantes (Coords.), Investigaciones en comportamiento alimentario. Reflexiones, alcances $y$ retos (pp. 13-59) Porrúa. http://www.cusur.udg.mx/es/sites/default/files/investigaciones_en_com portamento_alimentario.pdf

[11] Villalobos, A., Unikel, C., Hernández-Serrato M. I., \& Bojórquez I. (2020). Evolución de las conductas alimentarias de riesgo en adolescentes mexicanos, 2006-2018. Salud Publica de México, 62(6), 734-744. https://doi.org/10.21149/11545

[12] Asociación Americana de Psiquiatría (2014). Manual Diagnostico y Estadístico de los Trastornos Mentales DSM-5 ( $5^{\mathrm{a}} \mathrm{ed}$.). Editorial Médica Panamericana. https://1lib.mx/book/5898752/84fbf4

[13] Unikel-Santoncini, C., Bojórquez-Chapela, I., Villatoro-Velázquez, J., Fleiz-Bautista, C., \& Medina-Mora, M. I. (2006). Conductas alimentarias de riesgo en población estudiantil del Distrito Federal: tendencias 1997-2003. Revista de investigación clínica, 58(1), 15-27. http://www.scielo.org.mx/scielo.php?script=sci arttext\&pid=S0034$\underline{83762006000100003 \& \operatorname{lng}=e s \& t \operatorname{lng}=e s}$

[14] Buitrago, F., Tejero, M., \& Pagador, A. (2019). Trastornos de la conducta alimentaria y de la ingestión de alimentos. Actualización en Medicina de Familia, 15(1), 4-14. https://amfsemfyc.com/web/article_ver.php?id=2364

[15] Altamirano, M. B., Vizmanos, B., \& Unikel, C. (2011). Continuo de conductas alimentarias de riesgo en adolescentes de México. Revista Panamericana de Salud Pública, 30(5), 401-407. https://www.scielosp.org/pdf/rpsp/2011.v30n5/401-407/es

[16] Ortega-Luyando, M., Amaya-Hernández, A., Álvarez-Rayón, G., Vázquez-Arévalo, R., \& Mancilla-Díaz, J. M. (2018). Prevalencia de las conductas de riesgo. Un análisis de dos décadas. En A. López-Espinoza, A. G. Martínez \& V. G. Aguilera-Cervantes (Coords.), Investigaciones en comportamiento alimentario. Reflexiones, alcances y retos (pp. 129164) Porrúa. http://www.cusur.udg.mx/es/sites/default/files/investigaciones_en_com portamento_alimentario.pdf

[17] Fairburn, C. (2017). La superación de los atracones de comida. Cómo superar el control. (2da. ed.) Ediciones Paidós. https://pladlibroscl0.cdnstatics.com/libros_contenido_extra/36/35713 LA_SUPERACION_DE_LOS_ATRACONES.pdf

[18] Astolfo, M. A., Olivera, N. M., Pongelli, V., Fernández, N., \& Olmos, V. (2019). Uso inapropiado de diuréticos. Algunas características no tan conocidas. Acta Toxicológica Argentina, 27(1), 5-12. https://toxicologia.org.ar/wp-content/uploads/2019/06/Astolfo-finalOK.pdf

[19] Alvarado, A. M., Guzmán, E., \& González, M. T. (2005). Obesidad: ¿baja autoestima? intervención psicológica en pacientes con obesidad. Enseñanza e Investigación en Psicología, 10(2), 417-428. http://www.redalyc.org/articulo.oa?id=29210214

[20] Lameiras, M., Calado, M., Rodríguez, Y., \& Fernández, M. (2003). Hábitos alimentarios e imagen corporal en estudiantes universitarios sin trastornos alimentarios. International Journal of Clinical and Health $\begin{array}{lll}\text { Psychology, } & 3(1), & 23-33 .\end{array}$ https://www.redalyc.org/pdf/337/33730102.pdf

[21] Franco, K., Martínez, A. G., Díaz, F., Lopez-Espinoza, A., Aguilera, V., \& Valdés, E. (2010). Conductas de riesgo y sintomatología de trastornos del comportamiento alimentario en estudiantes universitarios del Sur de Jalisco, México. Revista Mexicana de Trastornos Alimentarios,

1 , 90-101. 
http://www.scielo.org.mx/scielo.php?script=sci_arttext\&pid=S2007. $\underline{15232010000200002}$

[22] Ramón, E., Martínez, B., Granada, J. M., Echániz, E., Pellicer, B. Juárez, R., Guerrero, S., \& Sáez, M. (2019). Conducta alimentaria y su relación con el estrés, la ansiedad, la depresión y el insomnio en estudiantes universitarios. Nutrición Hospitalaria, 36(6), 1339-1345. https://dx.doi.org/10.20960/nh.02641

[23] Serin, E., \& Can Koç, M. (2020). Examination of the eating behaviours and depression states of the university students who stay at home during the coronavirus pandemic in terms of different variables. Progress in Nutrition, 22(1), 33-43. https://doi.org/10.23751/pn.v22i1-S.9780

[24] Tazeoglu, A., Bozdogan, F., \& Idiz, C. (2021). Evaluation of Osmaniye Korkut Ata University Students' Eating Behaviors During the Quarantine Period during the COVID-19 Pandemic Period. Nutrición Clínica y Dietética Hospitalaria, 41(2), 86-93. https://doi.org/10.12873/412tazeoglu

[25] Almendra-Pegueros, R., Baladia, E., Ramírez-Contreras, C., RojasCárdenas, P., Vila-Martí, A., Moya, J., Apolinar-Jiménez, E., LazzaraLópez, A., Buhring-Bonacich, K., Nessier, M. C., Martínez-Vázquez, S., Camacho-López, S., Zambrana-Vera, A., Martínez-López, P., Raggio, L. M., \& Navarrete-Muñoz, E. V. (2021). Conducta alimentaria durante el confinamiento por COVID-19 (CoV-Eat Project): protocolo de un estudio transversal en países de habla hispana. Revista de Nutrición Clínica y Metabolismo, 4(3), 150-156. https://doi.org/10.35454/rncm.v4n3.267

[26] Pérez-Rodrigo, C., Gianzo, M., Hervás, G., Ruiz, F., Casis, L., \& Aranceta, J. (2020). Cambios en los hábitos alimentarios durante el periodo de confinamiento por la pandemia COVID-19 en España. Revista Española de Nutrición Comunitaria, 26(2). https://doi.org/10.14642/RENC.2020.26.2.5213 